\title{
UV Upturn in Elliptical Galaxies: Theory
}

\author{
Sukyoung K. Yi and Suk-Jin Yoon \\ University of Oxford, Astrophysics \\ Published in 2004,Astrophysics and Space Sciences, 291, 205
}

\begin{abstract}
I review the current theoretical understanding on the UV upturn phenomenon in early-type galaxies.
\end{abstract}

Keywords: UV upturn, Galaxy Evolution, sdB stars, EHB stars

\section{History}

The UV upturn is the rising flux with decreasing wavelength between the Lyman limit and $2500 \AA$ found virtually in all bright spheroidal galaxies. It has been a mystery ever since it was first detected by the OAO-2 space telescope (Code \& Welch 1979) because such old metal-rich populations were not expected to contain any substantial number of hot stars. It was confirmed by following space missions, ANS (de Boer 1982), IUE (Bertola et al. 1982) and HUT (Brown et al. 1997). The positive correlation between the UV-to-optical colour (i.e., the strength of the UV upturn) and the Mg2 line strength found by Burstein et al. (1987) through IUE observations has urged theorists to construct novel scenarios in which metal-rich $\left(\gtrsim Z_{\odot}\right)$ old $(\gtrsim$ a few Gyr) stars become UV bright (Greggio \& Renzini 1990; Horch et al. 1992). Also interesting was to find using HUT that, regardless of the

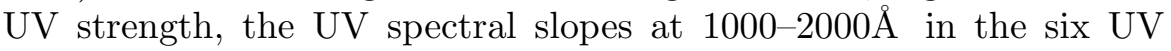
bright galaxies were nearly identical suggesting a very small range of temperatures of the UV sources in these galaxies (Brown et al. 1997), which corresponds to $T_{\text {eff }} \approx 20,000 \pm 3,000 \mathrm{~K}$. This, together with other evidence, effectively ruled out young stars as the main driver of the UV upturn. A good review on the observational side of the story is given in the next article by Tom Brown, as well as in the recent articles of Greggio \& Renzini (1999) and O'Connell (1999).

\section{Theory}

Theorists aim to present a model that explains three basic observational facts: (1) UV upturn being ubiquitous in the cores of bright elliptical galaxies whose optical stellar lights appear to be dominated by old and metal-rich stars, (2) positive correlation between the strength of

(C) 2018 Kluwer Academic Publishers. Printed in the Netherlands. 
the UV upturn and the optical metal line (Mg2) strength, and (3) a narrow range of temperature of UV sources.

\subsection{Metal-POOR HB Hypothesis}

It is widely known that metal-poor HB stars can be hot and make good UV sources when they are old (see the article by Yoon in this volume). Thus, the first scenario was naturally that bright elliptical galxies contain a fraction (perhaps order of $20 \%$ ) of stellar mass in the core in extremely old and metal-poor populations (Park \& Lee 1997). The strength of this scenario is that the oldest stars in a galaxy must also be the most metal-poor and are likely to be in the core, where the UV upturn is found. Besides, such old metal-poor HB stars are nothing too exotic to believe. In this scenario, the UV vs Mg2 relation does not present causality but simply a result of tracing different populations in terms of metallicity. The narrow range of temperature is very well reproduced as well. On the other hand, the mass fraction of order $20 \%$ is too high to be allowed by the standard galactic chemical evolution theory. If they are present at that level, there must also be a large number of intermediate-metallicity (20-50\% solar), which will make galaxy core's integrated metallicity too low and its integrated colours too blue, compared to observed values. Moreover, the age of the oldest stars, i.e. the main UV sources, must be $30 \%$ older than the average Milky Way globular clusters. Cosmology and stellar evolution theory (mainly, on globular clusters) have just reached a common ground where they approve each other in terms of the age of the universe. But now this scenario requires a large cosmological constant $\Omega_{\Lambda} \gtrsim 0.8$ and poses a problem to the apparent peace. This called for alternative scenarios.

\subsection{Metal-Rich HB hypothesis}

Stellar evolutionists found that metal-rich HB stars could in fact be more effective UV bright sources if galactic helium is enriched with respect to heavy elements at a rate of $\Delta Y / \Delta Z \gtrsim 2.5$ or if the mass loss rate in metal-rich stars is 30-40\% higher than that of metal-poor stars (Horch et al. 1992; Dorman et al. 1995; Yi et al. 1997a). Both of these conditions are difficult to validate empirically but quite plausible (Yi et al. 1998). In this scenario, metal-rich stars become UV bright in two steps: (1) they lose more mass on the red giant phase due to the opacity effect and become low-mass HB stars, and (2) extremely low-mass HB stars stay in the hot phase for a long time and directly become white dwarfs, effectively skipping the red, asymptotic giant phase (Yi et al. 1997a, 1997b). This scenario reproduces most of the features of the UV 
upturn (Bressan et al. 1994; Yi et al. 1998). The UV vs Mg2 relation is also explained. On the other hand, its validity heavily hinges upon the purely-theoretical late-stage stellar evolution models of metal-rich stars.

\subsection{WHICH THEORY?}

Both of these scenarios are equally appealing but their implications on the age of E galaxies are substantially different. The metal-poor hypothesis suggest UV- strong galaxies are 30\% older than the Milky Way (MW) and requires the universe to be older than currently believed, suggesting a large cosmological constant. The metal-rich hypothesis on the other hand suggests that E galaxies are not necessarily older than the Milky Way halo.

\section{Obsrvational tests}

The first obvious question is whether we can measure the mean metallicity of the UV sources from the high resolution UV spectra of the UVupturn galaxies. Brown et al. (2002) derived roughly 10\% solar from the HST/STIS data on NGC 1399 but found the derived value misleading because such highly-evolved hot HB stars do not show their intrinsic metallicities due to various heavy element redistribution processes. Spectroscopy does not seem promising at the moment.

Yi et al. (1999) presented a redshift-evolution test. Though both the metal-poor and metal-rich hypotheses are calibrated to the $z=0$ galaxies, they predict different evolutionary paths in the UV strength as a function of time. Thus, by reaching as far as $z \approx 0.3$, one might be able to select the more likely model. Figure 1(a) shows their qualitative prediction. Brown et al. $(1998,2000,2003)$ obtained UV data on three galaxy clusters at $z=0.33,0.375,0.55$. Figure $1(\mathrm{~b})$ shows them compared to our metal-rich HB hypothesis models. The models are denoted by the shaded region defined by the spread in the metallicity by a factor of two (the more metal-rich models, the more UV-strong). The earliest data on the $z=0.375$ cluster (shown as triangles) appear too red to be fit by the models and show no significant evolution in the UV flux from $z=0$, which surprised many theorists (including me). The more recent two cluster data with proper error estimates however look consistent with the metal-rich HB hypothesis models. Brown at this meeting cautiously stated that the first data set at $z=0.375$ obtained using HST/FOC may be dubious. It is encouraging to see the models mimic the two cluster data reasonably well, but we definitely need more data. 


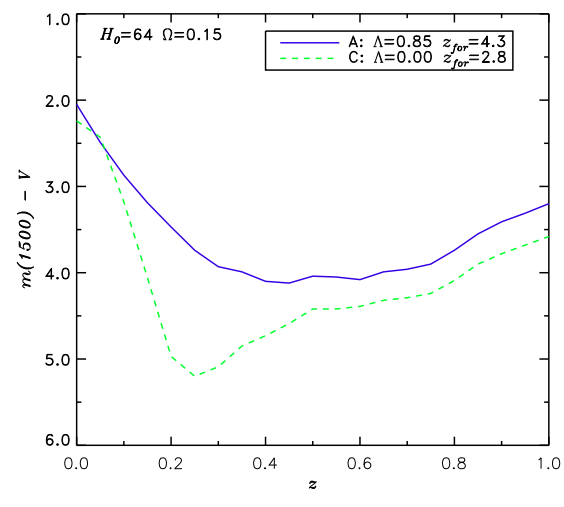

a. predicted $z$-evolution

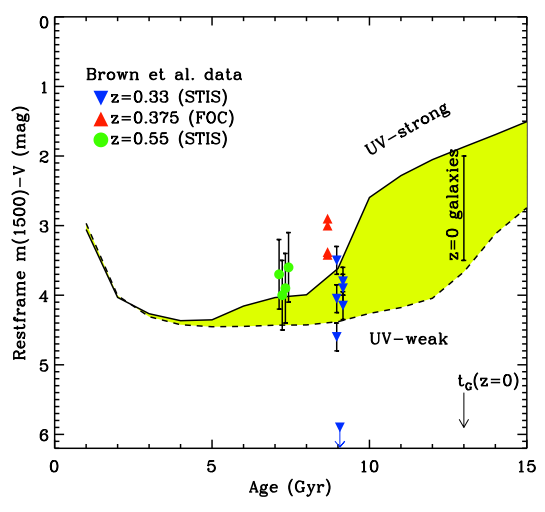

b. comparison with data

Figure 1. (a) The two representative UV evolution models from Yi et al. (1999). Model A: metal-poor HB (older) hypothesis, Model C: metal-rich HB (younger) hypothesis. (b) The HST data (triangles are now considered unreliable) seem consistent with the models (here shown are metal-rich models), but more data are required.

\section{Issues}

Readers may get an impression by reading the previous sections that we have solid and successful theories. Quite contrarily, there are several critical issues to be understood before we can ever claim so.

\section{1. $\alpha$-ENHANCEMENT}

Theorists (including myself) often interpret the UV vs Mg2 relation as a metallicity effect on the UV flux. However, it should be noted that Mg2 strength may not be representative of the overall metallicity. In fact, it has been known that elliptical galaxies are enhanced in $\alpha$-elements with respect to iron. We then naturally wonder if it is not the overall metallicity but $\alpha$-enhancement that generates the UV upturn. To perform this test, we need $\alpha$-enhanced stellar models. The $Y^{2}$ Isochrones group have released their stellar models for the main sequence (MS) through red giant branch (RGB) (Yi et al. 2003, see also my another article in this volume). No $\alpha$-enhanced HB models are available yet. $\alpha$ enhancement can have several impacts on the galaxy spectral evolution. First, it changes the stellar evolutionary time scale, as CNO abundance affects the nuclear generation rates. Second, it changes opacities and thus the surface temperatures of stars. These two effects will make a change in the mass loss computed using a parameterised formula, such as the Reimers formula. For a fixed mass loss efficiency, we find the $\alpha$-enhanced $([\alpha / \mathrm{Fe}]=0.3-0.6)$ tracks yield $\approx 0.03 M_{\odot}$ smaller mass 


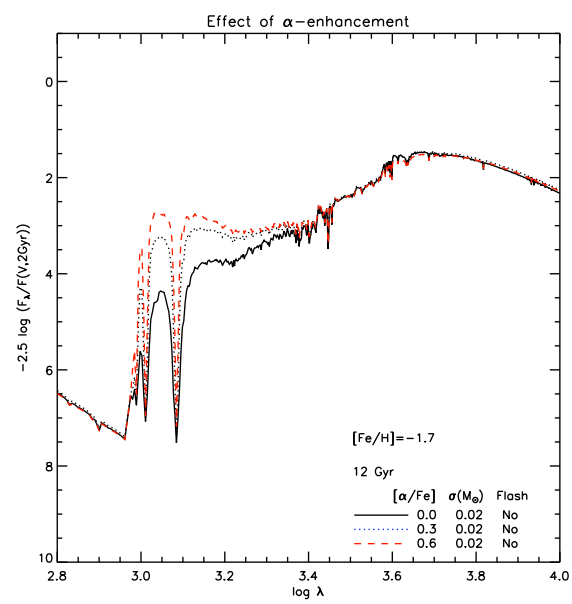

a. predicted $z$-evolution

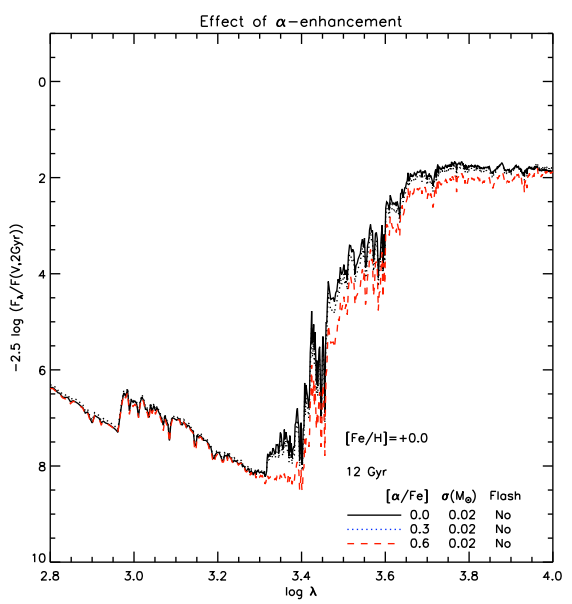

b. comparison with data

Figure 2. (a) The two representative models from Yi et al. (1999). Model A: metal-poor HB (older) hypothesis, Model C: metal-rich HB (younger) hypothesis. The two models predict different $z$-evolution. (b) The HST data (triangles are now considered unreliable) seem consistent with the metal-rich models, but more data are required.

loss at ages $5-8 \mathrm{Gyr}$ but $\approx 0.03 M_{\odot}$ greater mass loss at ages $\gtrsim 8 \mathrm{Gyr}$, compared to the standard $([\alpha / \mathrm{Fe}]=0)$ tracks. $\alpha$-enhancement must have similar opacity effects on the HB evolution except that mass loss on the HB is negligible. Thus its effects are expected to be greater to the MS to RGB than to the HB phase. Considering all these, I decided to inspect the overall effects of $\alpha$-enhancement by just adopting new $\alpha$-enhanced MS through RGB tracks, ignoring the change in the HB models. Figure 2 shows the results for two metallicities and three values of $\alpha$-enhancement. In old metal-poor model (Fig. 2(a)) $\alpha$-enhancement causes a positive effect to the relative UV strength because (1) it causes a slight increase in mass loss on the RGB and (2) it causes MS stars and red giants to be redder and fainter in $V$ band. The $[\alpha / \mathrm{Fe}]=0.3$ model roughly reproduces the SED of a typical UV-strong metal-poor globular cluster, which is quite satisfying. The metal-rich models (b) do not show any appreciable change in response to $\alpha$-enhancement. Because giant E galaxies are largely metal-rich (roughly solar) and the light contribution from metal-poor stars even in the core is not substantial, this exercise by itself does not seem to support the idea that $\alpha$-enhancement is the main driver of the UV upturn. 


\subsection{EHB STARS IN GLOBUlar CLUSTERS}

With the HST spatial resolution, a number of studies have found hot, extended horizontal branch (EHB) stars in globular clusters (e.g., Piotto et al. 1999). They are efficient UV sources and could be important candidates as the main UV sources in E galaxies; but there is no population synthesis model that successfully reproduces them as they are observed (number density, colours and brightness).

\subsection{NGC 6791}

This old (8-9Gyr) metal-rich (twice solar) cluster is unique for being close to the stellar populations of the E galaxy cores. Strikingly, roughly 9 out of its $32 \mathrm{HB}$ stars have the properties of typical EHB stars (Kaluzny \& Udalski 1992; Liebert et al. 1994). Figure 3(a) shows the well-defined red clump (compared to a synthetic HB shown as diamonds) and several hot (probably EHB) stars shown in box (see Yong et al. 2000 for details). Note that the synthetic HB does not contain any hot HB stars, that is, no diamond away from the clump. It is critical to understand the origin of these hot old metal-rich stars. Landsman et al. (1998), based on UIT data, concluded that NGC 6791, if observed from afar without fore/background stellar contamination, would exhibit a UV upturn just like the ones seen in E galaxies.

Through detailed synthetic HB modelling I have found that it is impossible to generate an $\mathrm{HB}$ with such a severely-bimodal colour distribution as shown in this cluster, unless an extremely (and unrealistically) large mass dispersion is adopted. In the hope of finding a mechanism that produces such an HB Yong et al. (2000) explored the effect of mass loss on the HB. Yong et al. found that with some mass loss taking place on the HB $\left(\approx 10^{-9}-10^{-10} M_{\odot} y r^{-1}\right)$ HB stars born cool quickly become hot, suggesting that mass loss on the HB might be an effective mechanism of producing such stars. Figure 3(b) shows a sample result. The top panel shows the observed colour distribution, red clump stars being simply put as an asterisk. The middle panel shows a synthetic HB assuming $10^{-9} M_{\odot} y r^{-1}$, with a Reimers mass loss efficiency $\eta=1(\approx 40 \%$ greater than that derived from metalpoor globular cluster stars) and a gaussian mass dispersion parameter $\sigma=0.04 M_{\odot}$. The bottom panel compares the distributions from theory and observation; the agreement is quite good. Do we have a convincing theory? If so, what causes this mass loss?

Vink \& Cassisi (2002) however pointed out that the scale of the mass loss assumed by Yong et al. is not justified by their radiation pressure calculations. It seems that such mass loss is not supported in single

stellar evolution. Green et al. (2000) reported that most of these hot 


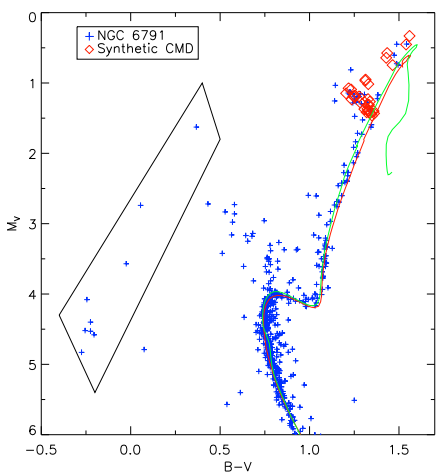

a. NGC 6791

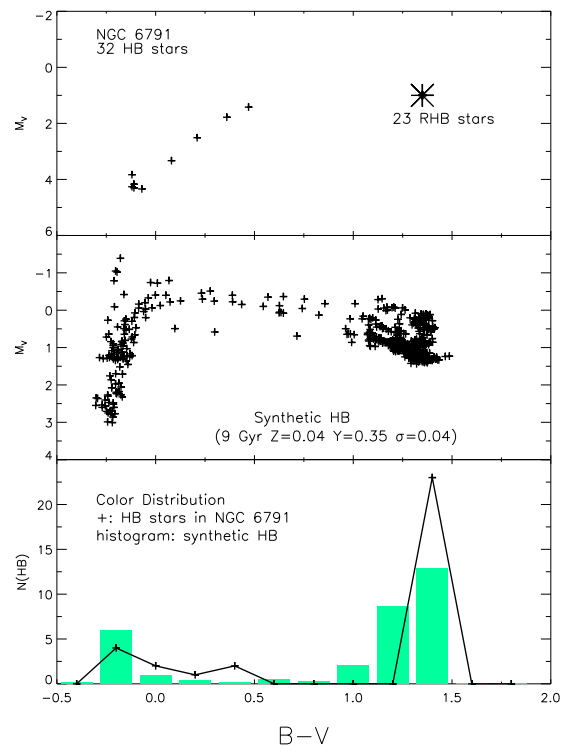

b. Colour distribution

Figure 3. (a) NGC 6791. The hot stars (shown in box) are not reproduced by canonical models (diamonds). (b) With mass loss assumed to take place on the HB the colour bimodality is reproduce.

stars in NGC 6791 are in binary systems. If they are close binaries and experience mass transfer it would be an effective mechanism for mass loss on the HB as well as on the RGB.

\subsection{BinARIES}

SdB stars may be the field counterparts of the EHB stars in clusters. They also have the properties that are similar to those of the UV sources in the UV-upturn galaxies. More than $70 \%$ of sdB stars are found to be in binary systems (Saffer et al. 2000; Maxted et al. 2001), and we may be looking at a smoking gun.

Han et al. (2003) used binary population synthesis technique to study the effects of binary evolution and found that $75-90 \%$ of sdB stars should be in binaries. SdBs are detected to be in a small mass range centered at $0.5 M_{\odot}$, but Han et al. found that the range should be in truth as wide as 0.3 through $0.8 M_{\odot}$. They predict a birthrate of $0.05 \mathrm{yr}^{-1}$ for Population I stars and 6 million sdB stars in the disc. Assuming the Galactic Disc mass of $5 \times 10^{10} M_{\odot}$, this means roughly $100 \mathrm{sdB}$ stars per $10^{6} M_{\odot}$. In a back-of-the-envelope calculation, there are roughly a few thousand HB stars per million solar mass in globular 
cluster populations. The comparison between the sdB rate (100 per $10^{6} M_{\odot}$ ) and that of the HB (say, 5000 per $10^{6} M_{\odot}$ ) suggests an old disc population may develop $1 \mathrm{sdB}$ star for $50 \mathrm{HB}$ stars $(2 \%)$. This is hardly impressive. NGC 6791 shows roughly $30 \%$ and the most UVbright globular cluster $\omega$ Cen $20 \%$. Yet, even $\omega$ Cen does not exhibit a UV upturn. If this calculation is realistic at least within an order, binary mass transfer may not provide the origin of the majority of the UV sources in UV-upturn galaxies. On the other hand, a larger sdB production rate might be plausible in elliptical galaxy environment due to large age and/or large metallicity. A more detailed study is very tempting.

\subsection{OTHER ISSUES}

There are other important issues as well. For example, the late-stage flash mixing scenarios and the like (D'Cruz et al. 1996; Brown et al. 2001) may also be effective ways of producing hot stars (such as sdB stars) in old populations. Their typical temperature range and the predicted birthrate may not be entirely consistent with the UV upturn shown in E galaxies, however.

Another important observational constraint comes from the HST UV images of M32. First, Brown et al. (2000) found that PAGB stars are two orders of magnitudes fewer than predicted by simple stellar evolution theory. This is significant as PAGB stars may contribute 10$30 \%$ of the UV flux in the UV-upturn galaxies. More importantly, they find too many hot $\mathrm{HB}$ stars to reproduce with standard population models that are based on the mass loss rate calibrated to the globular cluster HB morphology $(\eta \approx 0.65)$. It is possible to reproduce the observed number of hot stars in M32-type populations if a greater mass loss rate is used, which would be consistent with the variable mass loss hypothesis of Yi et al. (1997b, 1998).

\section{Summary}

The UV upturn provides tests of various aspects of stellar evolution and constrains the ages of elliptical galaxies. The current population synthesis models reasonably reproduce UV to optical broadband colours of current epoch galaxies, but there are multiple solutions. Redshift evolution observations have been proposed to differentiate these models. The HST/STIS instrument is being used effectively (Brown and

collaborators) and the GALEX (Martin et al. 1998) has a program to explore this subject. 
As soon as we look at the observed UV continua (spectra rather than colours) and the models, we realise something is not quite right with the models yet. The detailed spectral shape comparison clearly shows that there should be a stronger temperature bimodality in the HB than current models suggest. This seems related with the colour bimodality in the HB and the presence of the EHB stars in globular clusters found in many star clusters. The origin of sdB stars may also provide a clue. GALEX All Sky Survey will detect nearly all sdB stars within $25 \mathrm{kpc}$ at Galactic latitude of roughly 30 or greater. The data may pin down the number density and birthrate of sdB stars further. A more fine tuning on the mass loss calibration, as evident from M32 observations, seems required as well. Regarding the composite nature of galaxies, the colour bimodality may also very well arise from a large age difference between metal-poor (older) and metal-rich (younger) populations. When we have a better understanding on these issues, we will be able to find a convincing theory at last.

\section{Acknowledgements}

I thank the Keele Conference Local Organising Committee for the exciting meeting (with ample discussion) and for inviting me to be part of the Science Organising Committee and deliver this talk.

\section{References}

Bressan, A., Chiosi, C., \& Fagotto, F. 1994, ApJS, 94, 63

Brown, T. M., Ferguson, H. C., \& Davidsen, A. F. 1995, ApJ, 454, L15

Brown, T. M., Ferguson, H. C., Davidsen, A. F., \& Dorman, B. 1997, ApJ, 482, 685

Brown, T. M., Ferguson, H. C., Stanford, S. A., \& Deharveng, J.-M. 1998, ApJ, 504, 113

Brown, T. M., Bowers, C. W., Kimble, R, A., \& Ferguson, H. C. 2000, ApJ, 529, L89

Brown, T. M., Sweigart, A. V., Lanz, T., Landsman, W. B. \& Hubeny, I. 2001, ApJ, 562,368

Brown, T. M., Ferguson, H. C., Smith, E., Bowers, C. W., Kimble, R, A., Renzini, A., \& Rich, R. M. 2003, ApJ, 584, L69

Burstein, D., Bertola, F., Buson, L. M., Faber, S. M., \& Lauer, T. R. 1988, ApJ, 328,440

D'Cruz, N. L., Dorman, B., Rood, R. T. \& O'Connell, R. 1996, ApJ, 466, 359

Dorman, B., O'Connell, R., \& Rood, R. T. 1995, ApJ, 442, 105

de Boer, K. 1982, A\&AS, 50, 247

Green, E. M. et al. 2000, in the Third Faint Blue Star Conference, ed. D. Davis Philip, 333

Greggio, L. \& Renzini, A. 1999, Mem. S.A.It., 70, 691 
Greggio, L., \& Renzini, A. 1990, ApJ, 364, 35

Han, Z., Podsiadlowski, Ph., Maxted, P. F. L. \& March, T. R. 2003, 341, 669

Horch, E., Demarque, P., \& Pinsonneault, M. 1992, ApJ, 388, L53

Kaluzny, J. \& Udalski, A. 1992, AcA, 42, 29

Landsman, W., Bohlin, R. C., Neff, S. G., O'Connell, R. W., Roberts, M. S., Smith, A. M., \& Stecher, T. P. 1998, 116, 789

Liebert, J., Saffer, R. A., \& Green, E. M. 1994, AJ, 107, 1408

Martin, C. et al. 1998, BAAS, 29, 1309

Maxted, P. F. L., Heber, U., Marsh, T. R., \& North, R. C. 2001, MNRAS, 326, 1391

O'Connell, R. W. 1999, ARAA, 37, 603

Park, J.-H., \& Lee, Y.-W. 1997, 476, 28

Piotto, G., Zoccali, M., King, I. R., Djorgovski, S. G., Sosin, C., Rich, R. M., \& Meylan, G. 1999, AJ, 118, 1727

Reimers, D. 1975, Mém. Soc. Roy. Sci. Liège, 6th Ser., 8, 369

Saffer, R. A. et al. 2000, in the Third Faint Blue Star Conference, ed. D. Davis Philip, 444

Willson, L. A., Bowen, G. H., \& Struck, C. 1996, in ASP Series 98, From Stars to Galaxies, eds. C. Leitherer, U. Fritze-v. Alvensleben, \& J. Huchra (ASP), 197

Yi, S., Demarque, P., \& Kim, Y.-C. 1997a, ApJ, 482, 677

Yi, S., Demarque, P., \& Oemler, A. Jr. 1997b, ApJ, 486, 201

Yi, S., Demarque, P., \& Oemler, A. Jr. 1998, ApJ, 492, 480

Yi, S., Lee, Y.-W., Woo, J.-H., Park, J.-H., Demarque, P., \& Oemler, Jr. A. 1999, ApJ, 513, 128

Yi, S., Kim, Y.-C., \& Demarque, P., 2003, ApJS, 144, 259

Yong, H.-J., Demarque, P., \& Yi, S. 2000, ApJ, 539, 928 